\title{
Improving the Performance of Evolutionary Algorithms for the Multiobjective 0/1 Knapsack Problem Using $\varepsilon$-Dominance
}

\author{
Crina Groşan and Mihai Oltean \\ Department of Computer Science \\ Faculty of Mathematics and Computer Science \\ Babeş-Bolyai University, Kogălniceanu 1 \\ Cluj-Napoca, 3400, Romania. \\ \{cgrosan, moltean\}@cs.ubbcluj.ro
}

\begin{abstract}
The multiobjective 0/1 knapsack problem is a generalization of the $0 / 1$ knapsack problem in which multiple knapsacks are considered. A new evolutionary algorithm for solving multiobjective $0 / 1$ knapsack problem is proposed in this paper. This algorithm used a $\varepsilon$-dominance relation for direct comparison of two solutions. Several numerical experiments are performed using the best recent algorithms proposed for this problem. Experimental results clearly show that the proposed algorithm outperforms the existing evolutionary approaches for this problem.
\end{abstract}

\section{Introduction}

The 0/1 knapsack problem is a widely studied problem due its practical importance. In the last years a generalization of this problem was well studied and many algorithms for solving this variant have been proposed. Of great interest are the evolutionary approaches for solving the multiobjective $0 / 1$ knapsack problem. Many papers on the multiobjective knapsack problem and on the algorithms proposed for solving it can be found in the literature [12|3]. In this paper, we propose a new evolutionary approach for multiobjective $0 / 1$ knapsack problem. We use the $\varepsilon$-dominance concept which is a generalization of the standard Pareto concept.

In section 2 of the paper the multiobjective $0 / 1$ knapsack problems are presented. The description of the newly proposed algorithm is given in section 3 of the paper. The definition of $\varepsilon$-dominance concept is also given in section 3 Some comparisons with the most recent algorithms (such as SPEA2, NSGA II, PESA) are performed in section 4 .

\section{Problem Statement}

The classical 0/1 knapsack problem can be formulated as follow: a set of $n$ items and a knapsack of capacity $c$ are considered. Each item has a profit $p_{j}$ and a 
weight $w_{j}$. The problem is to select a subset of the items whose total weight does not exceed knapsack capacity $c$ and whose total profit is maximum.

The problem can be extended for an arbitrary number of knapsacks. A set of $\mathrm{n}$ items and a set of $\mathrm{k}$ knapsacks are considered. Each item has $k$ weight and $k$ profits corresponding to the each knapsack. Each knapsack has its own capacity. The objective is to select a subset of the items with maximum overall profit among those whose weight does not exceed the knapsack capacity for any of the $k$ dimensions.

\section{Proposed Algorithm}

The proposed algorithm uses $\varepsilon$-Pareto dominance relation between solutions.

We give here the definition of $\varepsilon$-Pareto dominance concept which is a generalization of the Pareto dominance concept.

\section{Definition}

Consider a maximization problem. Let $x, y$ be two decision vectors (solutions) from the search space. Solution $x \varepsilon$-dominate $y$ if and only if the following conditions are fulfilled:

$$
\begin{aligned}
& f_{i}(x) \geq f_{i}(y), \forall \quad i=1,2, \ldots, n, \\
& \exists j \in\{1,2, \ldots, n\}: f_{j}(x)>f_{j}(y)+\varepsilon .
\end{aligned}
$$

Each individual is a binary string. The value 1 for the position $j$ of the chromosome means that the item $j$ is selected to be included in a knapsack.

The proposed algorithm uses steady-state as its underlying mechanism and can be described as follows:

The algorithm starts with a population of randomly generated individuals. For each chromosome the total items weight for each knapsack is computed. If there are knapsacks for which the allowed capacity is exceeded the items starting with the one for which the proportion utility/weight has the smaller value are eliminated. This process continues until there are no knapsacks for which the capacity is exceeded. All nondominated solutions are computed using the $\varepsilon$-dominance concept. The following steps are repeated until a termination condition is reached: Two nondominates solutions (the parents) are randomly chosen. The parents are recombined using uniform crossover operator and the offspring are mutated. For each offspring the procedure for eliminating items if the capacity of one of knapsacks is exceeded is used. The offspring enters the population and the dominated solutions are removed.

This algorithm is called $\varepsilon$ Multiobjective Knapsack Algorithm ( $\varepsilon$-MOKA). 
Table 1. The values of $\varepsilon$ for the case of three knapsacks

\begin{tabular}{|l|l|l|}
\hline $\begin{array}{l}\text { Number of functions } \\
\text { evaluations }\end{array}$ & The value of $\varepsilon$ & $\begin{array}{l}\text { Number of generations after } \\
\text { which } \varepsilon \text { becomes } \varepsilon-1\end{array}$ \\
\hline 150,000 & 1000 & 500 \\
\hline 288,000 & 1000 & 1000 \\
\hline 576,000 & 1500 & 1000 \\
\hline
\end{tabular}

Table 2. The results obtained for three knapsacks by $\varepsilon$-MOKA, NSGA II, SPEA2 and PESA considering 150,000 functions evaluations. Results are averaged over 30 runs

\begin{tabular}{|l|l|l|l|l|}
\hline & $\varepsilon$-MOKA & NSGA II & SPEA 2 & PESA \\
\hline$\varepsilon$-MOKA & & 0.90301 & 0.939242 & 0.994266 \\
\hline NSGA II & 0 & & 0.976883 & 0.989555 \\
\hline SPEA 2 & 0 & 0.906996 & & 0.990861 \\
\hline PESA & 0 & 0.862837 & 0.908916 & \\
\hline
\end{tabular}

Table 3. The results obtained for three knapsacks by $\varepsilon$-MOKA, NSGA II, SPEA2 and PESA considering 288,000 functions evaluations. Results are averaged over 30 runs

\begin{tabular}{|l|l|l|l|l|}
\hline & $\varepsilon$-MOKA & NSGA II & SPEA 2 & PESA \\
\hline$\varepsilon$-MOKA & & 0.787402 & 0.910033 & 0.985815 \\
\hline NSGA II & 0 & & 0.996314 & 0.990203 \\
\hline SPEA 2 & 0 & 0.853698 & & 0.985883 \\
\hline PESA & 0 & 0.850779 & 0.93234 & \\
\hline
\end{tabular}

\section{Experimental Results}

We test our algorithm considering 750 items and three knapsacks. The results obtained by $\varepsilon$-MOKA are compared to the results obtained by SPEA2 [3], NSGA II 2 and PESA [1. For this comparison $C$ metric introduced by Zitzler is used. Test data are taken from [4].

Table 4. The results obtained for three knapsacks by $\varepsilon$-MOKA, NSGA II, SPEA2 and PESA considering 576,000 functions evaluations. Results are averaged over 30 runs

\begin{tabular}{|l|l|l|l|l|}
\hline & $\varepsilon$-MOKA & NSGA II & SPEA 2 & PESA \\
\hline$\varepsilon$-MOKA & & 0.58796 & 0.851949 & 0.931406 \\
\hline NSGA II & 0 & & 0.996237 & 0.991706 \\
\hline SPEA 2 & 0 & 0.832425 & & 0.96905 \\
\hline PESA & 0 & 0.809001 & 0.939329 & \\
\hline
\end{tabular}


Using $C$ metric two sets of nondominated solutions can be compared to each other. $C(A, B)$ return the proportion between number of solutions from $B$ which are dominated by solutions from $A$ over number of solutions from $B$. This number is a number between 0 and 1 . The value $C(A, B)=1$ means that all decision vectors in $B$ are dominated by $A$. The value $C(A, B)=0$ represent the situation when none of the points in $B$ are dominated by $A$.

General parameters used by the considered algorithms are: number of knapsacks: 3, population size: 300, number of functions evaluations: 150,000, 288,000, 576,000 .

The value of $\varepsilon$ for $\varepsilon$-MOKA is chosen as follows: at the beginning of the search process this value is a large one. In this way a low dominance is ensured and this allow us to preserve many solutions in the first generations. After a number of iterations the value of $\varepsilon$ is decreased by 1 . Reducing the value of $\varepsilon$ we will ensure the true Pareto dominance at the end of the search process.

The values of $\varepsilon$ are presented in Table 1 .

The results obtained by applying $C$ metric for 150,000, 288,000 and 576,000 are presented in Table 2, Table 3 and Table 4, respectively.

Solutions obtained by $\varepsilon$-MOKA are not dominated by solutions obtained by NSGA II, SPEA 2 and PESA even for 576,000 functions evaluation. This means $\varepsilon$-MOKA has a very good convergence.

\section{References}

1. Corne, D.W., Knowles, J.D.: The Pareto-Envelope based Selection Algorithm for Multiobjective Optimization. In Proceedings of the Sixth International Conference on Parallel Problem Solving from Nature, Springer-Verlag, Berlin (2000) 839-848

2. Deb, K., Agrawal, S., Pratap A., Meyarivan T.: A fast elitist non - dominated sorting genetic algorithm for multi-objective optimization: NSGA II. In: Shoenauer, M. et al. (eds), Parallel Problem Solving From Nature - PPSN VI, Springer-Verlag, Berlin (2000) 849-858

3. Zitzler, E., Marco Laumanns and Thiele, L.: SPEA 2: Improving the Strength Pareto Evolutionary Algorithm. TIK Report 103, Computer Engineering and Networks Laboratory (TIK), Departament of Electrical Engineering Swiss federal Institute of Technology (ETH) Zurich (2001)

4. http://www.tik.ee.ethz.ch/ zitzler/testdata.html 